\title{
Recurrence of adenoid cystic carcinoma of the trachea treated with radical radiotherapy: A case report
}

\author{
KONRAD PAWLEWICZ, ZBIGNIEW SZUTKOWSKI and ANDRZEJ KAWECKI \\ Department of Radiotherapy, The Maria Skłodowska Curie Memorial Cancer \\ Centre and Institute of Oncology, Warsaw 02-781, Poland
}

Received November 18, 2016; Accepted September 1, 2017

DOI: $10.3892 / \mathrm{ol} .2018 .7780$

\begin{abstract}
Adenoid cystic carcinoma (ACC) of the trachea is a relatively rare tumor among thoracic diseases. The present study reports the case of a 23-year-old woman with ACC of the trachea who underwent surgical resection of the tumor in The National Institute of Tuberculosis and Lung Diseases (Warsaw, Poland). Histopathological examination revealed that the tumor was not completely resected ( $\mathrm{R} 2$ resection) and strict observation of the patient was therefore prescribed. After $\sim 9$ years of follow-up, clinical and histopathological tumor progression was confirmed and the patient was referred to the Centre of Oncology in Warsaw. The localization and advanced nature of the disease precluded surgical intervention, and radical radiotherapy was therefore performed using intensity-modulated radiation therapy. A total dose of 7,590 Gy, the planning target volume, was administered. A hyperfractionation scheme of radiotherapy was used: 2 fractions of 1.15 Gy daily, with at least a $6 \mathrm{~h}$ break in between. Tumor regression was observed following treatment and has been maintained for $>3$ years, assessed by clinical and computed tomography and positron emission tomography imaging examinations.
\end{abstract}

\section{Introduction}

Adenoid cystic carcinoma (ACC) is a malignant neoplasm that derives from glandular tissue. ACC is most commonly located in the salivary glands, but it also occurs in other organs that contain glands, including the trachea (1). The occurrence of ACC in the trachea is extremely rare in Poland according to data from the Polish National Cancer Registry; in 2014, the incidence of tracheal cancer was 11 cases among the female population and

Correspondence to: Dr Zbigniew Szutkowski or Dr Konrad Pawlewicz, Department of Radiotherapy, The Maria Skłodowska Curie Memorial Cancer Centre and Institute of Oncology, 5 Roentgena Street, Warsaw 02-781, Poland

E-mail: zszutkowski@coi.pl

E-mail: konrad_pawlewicz@wp.pl

Key words: adenoid cystic carcinoma, tumor of the trachea, radical radiotherapy
18 cases among the male population, of which few were ACC (2). ACC is characterized by slow growth and local involvement; it exhibits a high tendency for recurrence. Symptoms result from obstruction of the tracheal lumen (3). Dyspnea, which increases over months, is the most common symptom (3). The primary management of tracheal ACC is surgical resection of part of the trachea and tumor with end-to-end anastomosis (3). Postoperative radiotherapy is recommended for patients that undergo incomplete resection (3). Radical radiotherapy should be considered as an option for patients with unresectable disease (3). The present study describes a case of recurrent ACC of the trachea in a young woman treated with irradiation.

\section{Case report}

A 23-year-old woman who had suffered from increasing dyspnea for months was admitted to the Thoracic Surgery Department of The Specialist District Hospital (Rzeszów, Poland) in December 2004 due to severe dyspnea accompanied by stridor. The patient reported that she had not experienced fever or weight loss. She had not suffered from a chronic disease, taken drugs, smoked cigarettes or abused alcohol.

An irregular tumor mass of $15 \times 21 \times 26 \mathrm{~mm}$ within the trachea at the level of tracheal bifurcation and slightly above and forward/anterior was visualized by a thoracic computed tomography (CT) scan. The tumor infiltrated the mediastinum anterior to the tracheal carina, affecting the area between the opening of the hemiazygos vein arch into the superior vena cava and aortic arch. The tumor penetrated into the tracheal lumen just above the bifurcation, medially narrowing the lumen to a $\sim 9 \mathrm{~cm}$ sagittal diameter. The patient did not present with pathologically enlarged lymph nodes in the mediastinum or hila of the lungs. A cauliflower-like tumor arising from the anterior wall and infiltrating 2-3 cm upward inside the trachea, $\sim 1 \mathrm{~cm}$ above its bifurcation, was visualized with bronchofibroscopy.

To restore tracheal patency, during rigid bronchoscopy the lesion was partially removed (R2 surgery). This was followed by a right-sided thoracotomy, during which the segment of the trachea with the tumor was resected and the paratracheal and subcarinal lymph nodes were removed.

Histopathological examination revealed ACC of the trachea. A neoplastic infiltration was present in the surgical margin. Metastases infiltrating and extending through the capsule were present in the lymph nodes. 
No adjuvant treatment was administered. The patient remained under close observation and a follow-up bronchofibroscopy was performed every 6 months. A polypous mucosal thickening, slightly narrowing the lumen of the bronchus, was visualized at the anastomosis site, particularly in the opening of the left primary bronchus, after 8.5 years in July 2013 . Specimens were collected by biopsy and a thoracic CT scan was performed. The CT scans revealed small nodular lesions arising from the wall towards the lumen in the region of the anastomosis, the lower part of the trachea, below the anastomosis and in the left primary bronchus, below the bifurcation. The lower segment of the trachea and short initial segments of the main bronchi were surrounded by a neoplastic infiltration between the division of the pulmonary artery and the site of the aortic arch branches, which exhibited contrast enhancement (Figs. 1 and 2). A recurrence was confirmed by histopathological examination. The patient was then admitted to The Maria Sklodowska Curie Memorial Cancer Centre and Institute of Oncology (Warsaw, Poland).

Surgical resection was not performed due to the extent of infiltration. Following assessment, the patient was recommended radiotherapy. For the purposes of treatment planning, CT and positron emission tomography (PET)-CT were performed. PET-CT revealed the presence of small tumors in the lumen of the trachea and the main bronchi, with an abnormal mass surrounding the trachea and its bifurcation exhibiting a slightly increased fludeoxyglucose metabolic rate, characteristic of ACC.

Hyperfractionated radiotherapy (2 fractions a day with at least a 6-h interval, 10 fractions a week) for 6.5 weeks was administered to the tumor, including the margin and mediastinum. The gross tumor volume (GTV) with a $1-\mathrm{cm}$ margin, including the mediastinal structures, was defined as the clinical target volume (CTV) area. The total dose of 7,590 cGy in fractions of $115 \mathrm{cGy}$ was administered to the GTV area and the total dose of 6,600 cGy in fractions of 100 cGy to the CTV area. The biologically effective dose (for an $\alpha / \beta$ ratio=10) to the tumor was $84.63 \mathrm{~Gy}$. The radiotherapy planning was based on a $6 \mathrm{MV}$ nine-field intensity-modulated radiation therapy (IMRT) technique (Fig. 3). The biological doses administered to critical organs were 1,326 cGy to the spinal cord and 1,685 cGy to the heart.

A CT scan following treatment exhibited a considerable regression of the neoplastic infiltration (Fig. 4). A PET-CT scan revealed no features of a metabolically active proliferative disease.

The patient remains under close follow-up. Imaging examinations (CT and PET-CT) are systematically performed every 6 months. The last PET-CT (May 2017), revealed no features of recurrence.

\section{Discussion}

ACC is a relatively rare malignant neoplasm that derives from glandular tissue. After squamous cell carcinoma, ACC is the second-most common trachea neoplasm, accounting for $30-40 \%$ of cases, according to data from the literature (3). The estimated incidence is $\sim 1$ case per million people, per year (3). ACC of the trachea is most commonly located in the distal third of the trachea in the region of its bifurcation. There is no evidence that cigarette smoking increases the risk of ACC (3). The incidence of ACC is the same for males and females, and usually occurs in the 4th or 5th decade of life (4). However, the case reported in the present study was diagnosed in a 23-year-old female patient.

Macroscopically, ACC is a poorly delimited solid tumor, usually in the form of polypous mucosal thickenings that decrease the lumen of the trachea. At an advanced stage, infiltration of the trachea wall and adjacent organs frequently occurs, exhibiting a tendency for submucosal dissemination and dissemination along nerves $(1,5,6)$. Long cylindrical structures surrounded by rectangular cells with hyperchromatic nuclei, characteristic of ACC, are visible under a microscope (1). These tumors metastasize into mediastinal lymph nodes and may metastasize to distant sites, most frequently into the lungs (7).

Analysis performed by Gaissert et al (8) demonstrated that the neoplasm infiltrated the tissues surrounding the trachea in $60 \%$ of all patients receiving surgery with ACC of the trachea, and adjacent organs, primarily the esophagus, in a further $15 \%$. Almost $60 \%$ were non-radical surgeries. Metastases into the lymph nodes were present in only $13 \%$ of patients undergoing surgical resection. This indicates the tendency for neoplasms to infiltrate into and along the tracheal mucosa, which predominates over metastasis into the lymph nodes (8).

The predominant symptoms of tracheal neoplasms result from the obturation of the tracheal lumen caused by the tumor. Dyspnea, increasing over recent months and later accompanied by stridor, is most often encountered. Other symptoms include whistling sounds, from the lower part of the bronchial tree, cough, chest pains and hemoptysis $(4,9)$. The mean time between the appearance of disease symptoms and the establishment of a diagnosis is long: 18.3 months in patients with neoplasms that can be resected and 23.7 months in patients with inoperable neoplasms (8). Patients are often treated for asthma for a substantial period of time prior to the establishment of an appropriate diagnosis (7). In the present case, the female patient presented with gradually increasing dyspnea and stridor, symptoms indicating upper airway obstruction. The peak expiratory flow index is reduced in patients with an obturation of the trachea, with a flat flow-volume curve (3).

Bronchoscopy and thoracic CTs are typically used to diagnose tracheal neoplasms. Abronchoscopic examination allows for the macroscopic assessment of local neoplasm progression and allows histological verification of the neoplasm. CT scans allow for the visualization of infiltration extent and metastases into the lymph nodes. On the basis of this examination, it is possible to create a virtual bronchoscopy. Chest X-rays are ineffective in the examination of tracheal neoplasms (1). Symptomatic management aims to restore tracheal patency during a rigid bronchoscopy. Such a procedure was performed in the present case.

Resection of the trachea and the tumor with end-to-end anastomosis or artificial trachea implantation at the defect site is the primary method of radical treatment. In cases where it is not possible to complete surgical resection, radiotherapy is administered to patients. ACC exhibits low radiation sensitivity and therefore the highest possible dose should be administered, taking the tolerance doses of adjacent organs (including the trachea, esophagus, spinal 

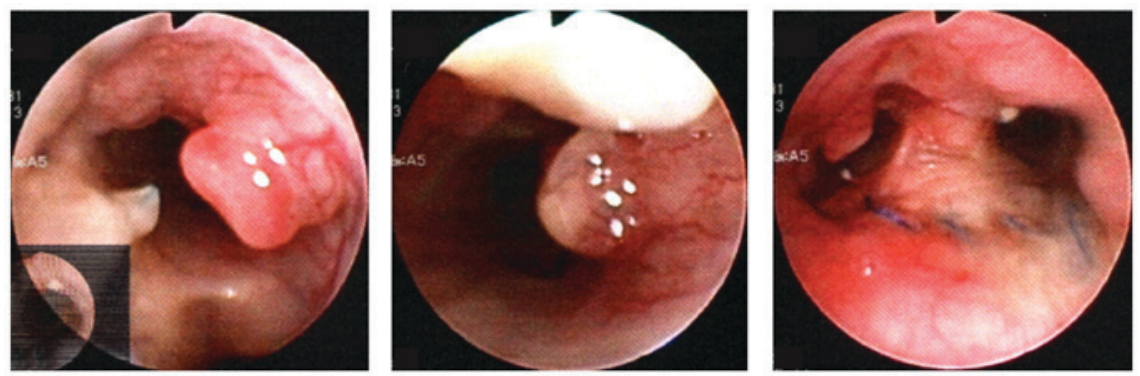

Figure 1. Bronchofibroscopy images, indicating polypous mucosal thickenings visible at the site of anastomosis.
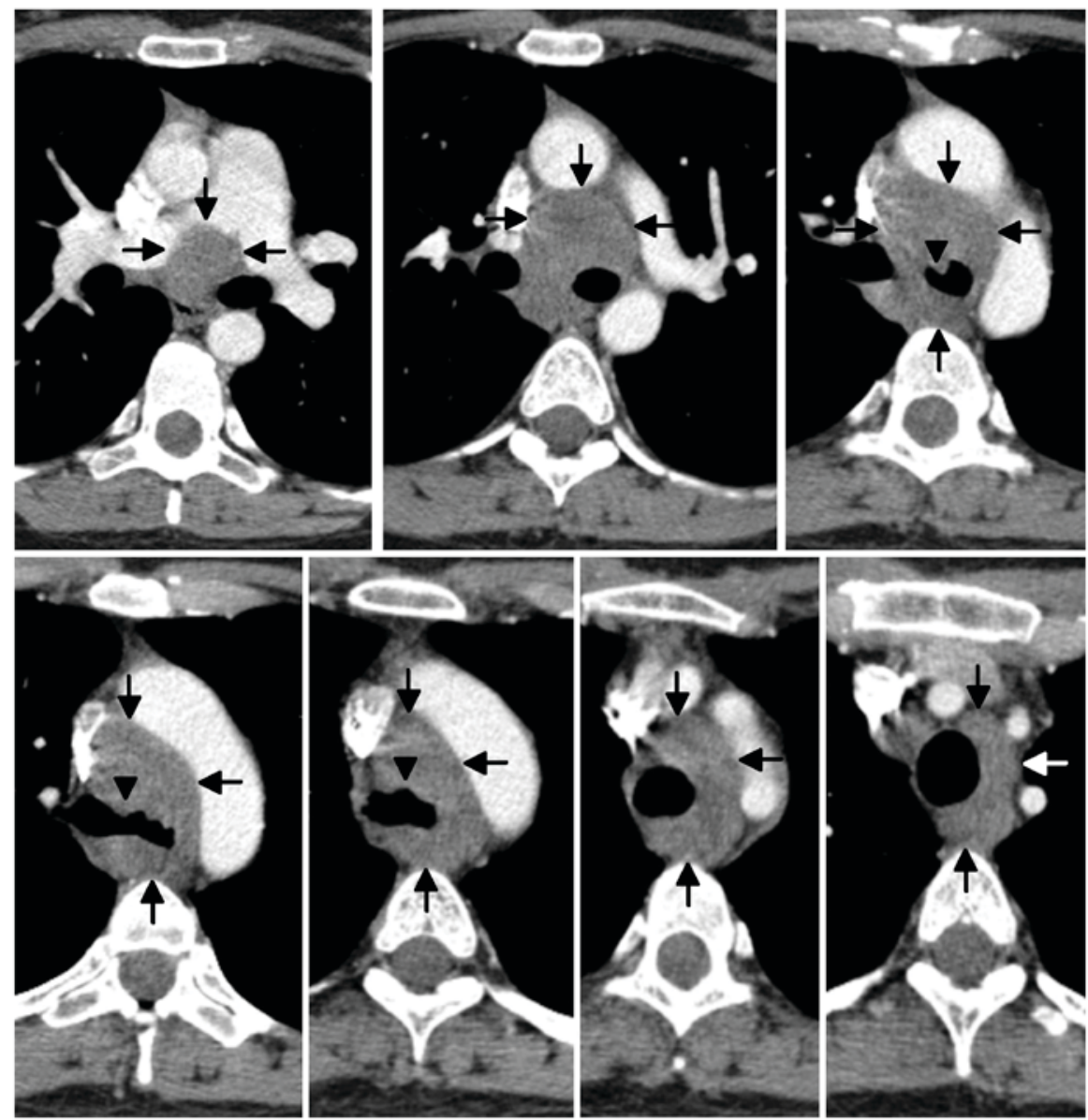

Figure 2. Thoracic computed tomography scans. Arrows indicate nodular lesions arising from the wall towards the lumen of the lower region of the trachea and left primary bronchus; arrowheads indicate the boundaries of infiltration.

cord, lungs and heart) into account. A dose of 70 Gy in fractions of 2 Gy administered once a day 5 days per week is indicated. Radiotherapy should be planned on the basis of conformal techniques, preferably with the use of IMRT (10). A previous study has proposed the possibility of combining teleradiotherapy with brachytherapy, boosting the radiotherapeutic dose to macroscopic disease areas and therefore limiting undesirable effects in adjacent critical tissues and organs $(10,11)$. Radiotherapy is shifting towards the use of proton therapies (12). The greater potential for sparing critical organs allows for an increase in the maximal doses of radiation. There is published study that presents the administration of doses of $80 \mathrm{~Gy}$ in fractions of $2 \mathrm{~Gy}$ without alterations to the tolerated doses for critical organs (12).
That the area at risk of microscopic disease has a tendency for submucosal infiltration and infiltration around nerves should be taken into account. Treating the upward and downward margins from neoplastic infiltration is recommended (7,10).

The role of postoperative radiotherapy is not clear. This procedure causes a decrease in local recurrence frequency; however, an increase in survival time has not previously been demonstrated. Metastasis into lymph nodes and a non-radical surgical procedure are obligatory indications for the use of postoperative radiotherapy $(3,13)$. A dose for the treatment of the microscopic disease should be at least $60 \mathrm{~Gy}$ in fractions of 2 Gy administered five times a week $(3,11)$. A higher dose should be given to residual disease areas and to surgical sites following 

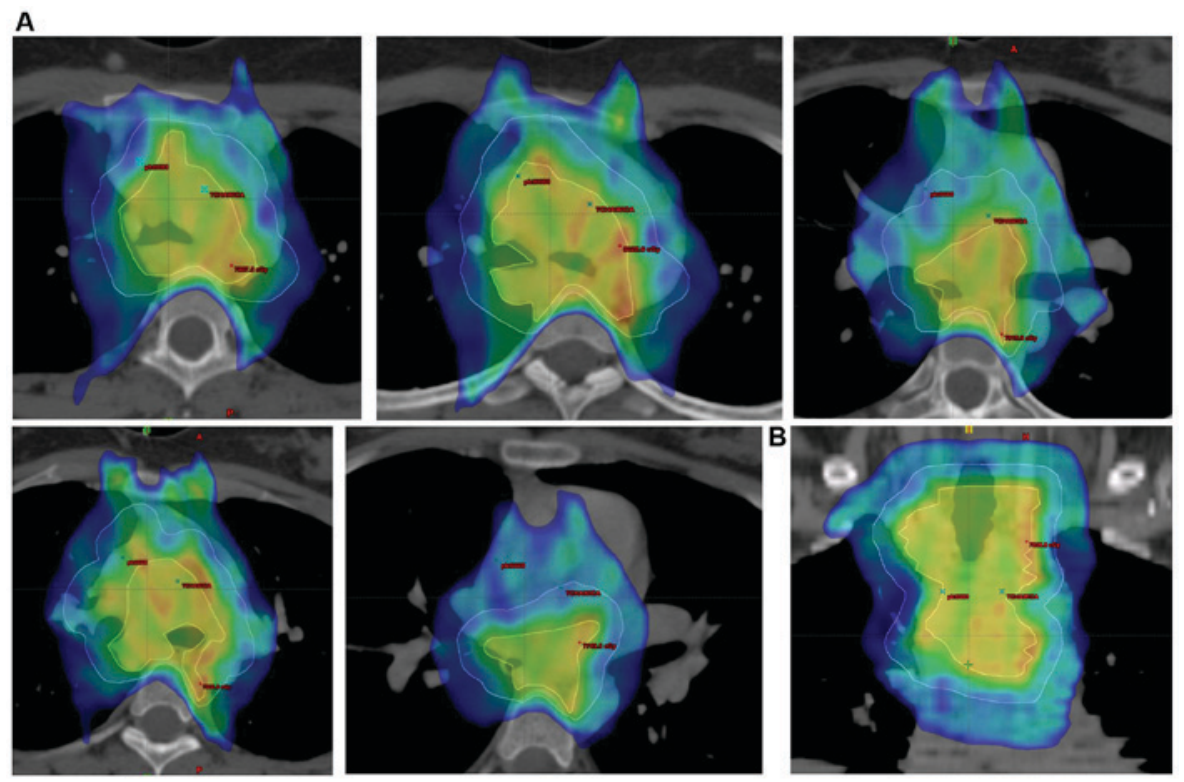

Figure 3. Radiotherapy plan. (A) Transverse cross-sections; (B) Frontal cross-section.
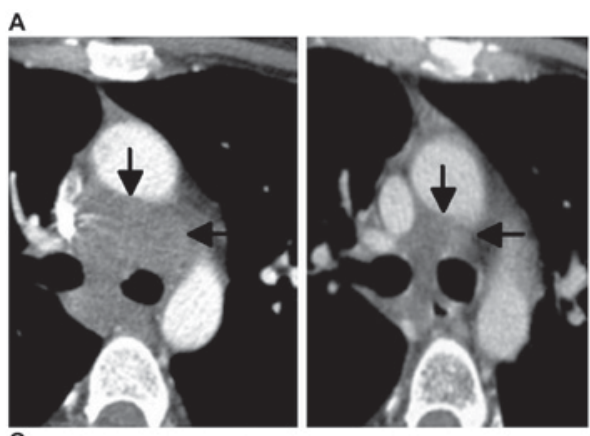

B
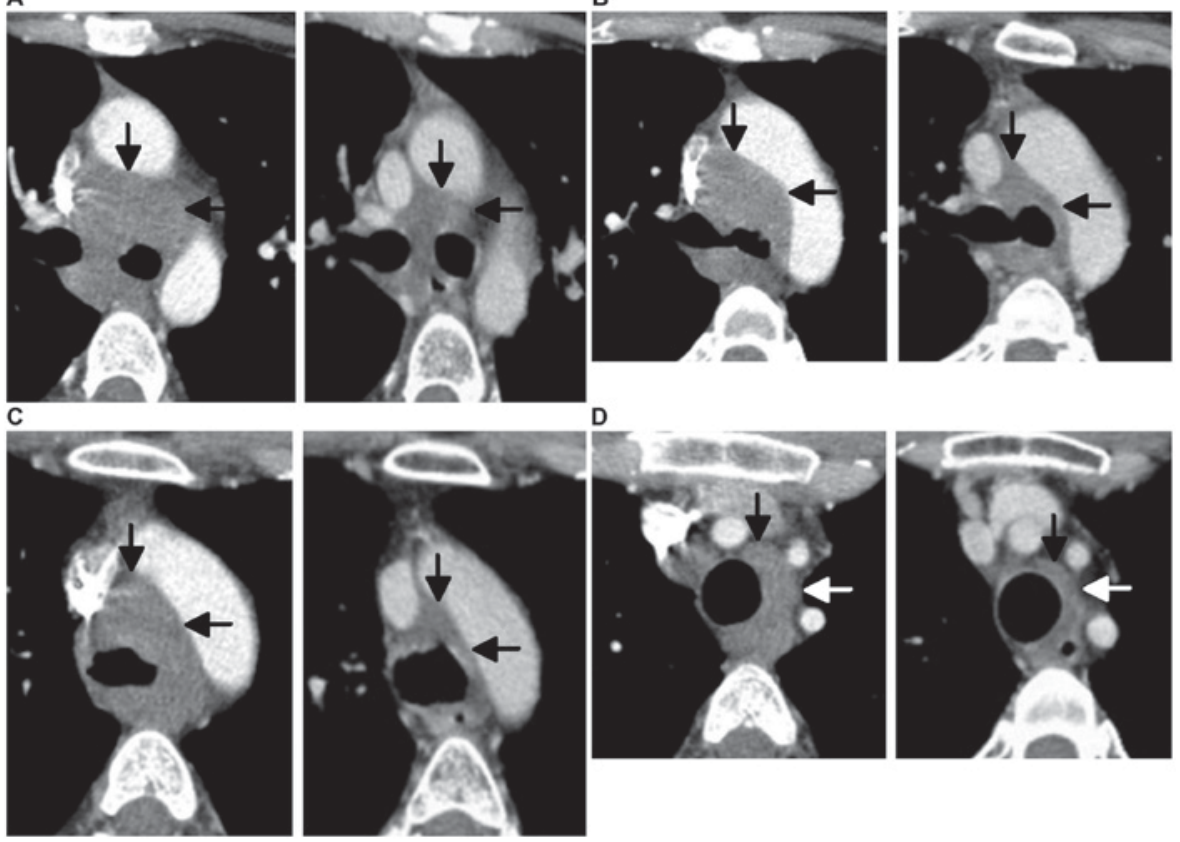

Figure 4. Assessment of treatment effectiveness. A comparison of CT scans prior to radiotherapy with CT scans subsequent to radiotherapy on the same level: (A) The level below the aortic arch, (B) the level of the carina, (C) the level of the aortic arch and (D) the level above the aortic arch. Arrows indicate the boundaries of infiltration prior to and following radiotherapy.

non-radical surgery (10). Postoperative radiotherapy planning should be conducted on the basis of CTs performed prior to the surgical procedure. Analysis performed by Chen et al (13) indicates that there is an improvement in disease-free and overall survival rates in patients who received postoperative radiotherapy following a non-radical procedure (13).

Chemotherapy is ineffective in the treatment of ACC of the trachea: Attempts to use chemotherapy in combination with radiotherapy or as palliative treatment have demonstrated no benefit (13).

The largest retrospective analysis of cases of ACC of the trachea was conducted at the Department of Thoracic
Surgery of Massachusetts General Hospital (Boston, MA, USA), including 135 patients from 1962-2002 (8). A total of $75 \%$ of patients underwent surgical resection. It was decided that surgery would not be performed in the remaining patients following assessment, due to the local progression of the disease. The authors emphasized that the increasing experience and novel technical possibilities in procedures, primarily restorative techniques, enabled surgery to be completed in patients with increasingly advanced neoplastic processes. Postoperative radiotherapy was performed for $70 \%$ of the patients that received surgery. The mean survival time was 69 months for patients that received surgery, and 
41 months for patients that did not. The 5-year survival rate was $54 \%$ in patients that received surgery and $33 \%$ in patients that did not; the 10 -year survival rates were 33 and $9 \%$, respectively (8).

Another analysis of the SEER medical database covered 94 cases of tracheal neoplasms from 1973 to 2004 (4). The data demonstrated that the 5-year overall survival rate of patients with ACC of the trachea was $74.3 \%$, whereas if this disease was diagnosed at a locoregionally advanced stage, the survival rate increased to $90 \%$ (4).

Data from the literature indicate a significant improvement of treatment outcomes following the use of surgical resection with adjuvant radiotherapy (13). In the described case, non-radical macroscopic surgery with subsequent close observation was not an optimal procedure. According to the present study, irradiation of late recurrences following prior $\mathrm{R} 2$ resection may enable a locoregional control to be achieved; however, the patient prognosis remains uncertain.

The aim of the present study was to indicate an atypical case following the first treatment in the local hospital, which contrasts with the regular practice in the Maria Skłodowska-Curie Memorial Cancer Center and Institute of Oncology. In this case the multidisciplinary team in an institution that offers the complete range of oncological treatment would make the therapeutic decisions.

\section{References}

1. Kwak SH, Lee KS, Chung MJ, Jeong YJ, Kim GY and Kwon OJ: Adenoid cystic carcinoma of the airways: Helical CT and histopathological correlation. AJR Am J Roentgenol 183: 277-281, 2004.
2. Wojciechowska U, Olasek P, Czauderna K and Didkowska J: Nowotwory złośliwe w polsce w 2014 roku. Cancer in Poland in 2014. Polish National Cancer Registry, Department of Epidemiology and Cancer Prevention, Warszawa, pp48-50, 2016 (In Poland).

3. Honings J, Gaissert HA, van der Heijden HF, Verhagen AF, Kaanders JH and Marres HA: Clinical aspects and treatment of primary tracheal malignancies. Acta Otolaryngol 130: 763-772, 2010.

4. Urdaneta AI, Yu JB and Wilson LD: Population based cancer registry analysis of primary tracheal carcinoma, Am J Clin Oncol 34: 32-37, 2011.

5. Choudhury BK, Barman G, Singh S and Ahmed K: Adenoid cystic carcinoma of the upper trachea: A rare neoplasm. J Clin Imaging Sci 3: 39, 2013

6. Vigg A, Mantri S, Vigg A and Vigg A: Adenoid cystic carcinoma of trachea. Indian J Chest Dis Allied Sci 46: 287-289, 2004.

7. El Marjany M, Arsalane A, Sifat H, Andaloussi K, Oukabli M, Hadadi K, Kabiri el $\mathrm{H}$ and Mansouri H: Primary adenoid cystic carcinoma of the trachea: A report of two cases and literature review. Pan Afr Med J 19: 32, 2014.

8. Gaissert HA, Grillo HC, Shadmehr MB, Wright CD, Gokhale M, Wain JC and Mathisen DJ: Long-term survival after resection of primary adenoid cystic and squamous cell carcinoma of the trachea and carina. Ann Thorac Surg 78: 1889-1896, 2004.

9. Yang PY, Liu MS, Chen CH, Lin CM and Tsao TC: Adenoid cystic carcinoma of the trachea: A report of seven cases and literature review. Chang Gung Med J 28: 357-363, 2005.

10. Choi NC: Radiation therapy in the management of tracheal cancer. In: Surgery of the trachea and bronchi. Grillo HC (ed). BC Decker, Hamilton, London, pp791-802, 2004.

11. Haresh KP, Prabhakar R, Rath GK, Sharma DN, Julka PK and Subramani V: Adenoid cystic carcinoma of the trachea treated with PET-CT based intensity modulated radiotherapy. J Thorac Oncol 3: 793-795, 2008.

12. Bonner Millar LP, Stripp D, Cooper JD, Both S, James P and Rengan R: Definitive radiotherapy for unresected adenoid cystic carcinoma of the trachea. Chest 141: 1323-1326, 2012.

13. Chen F, Huang M, Xu Y, Li T, Xie K, Zhang L, Cheng D, Liu L, Che G, Hou M, et al: Primary tracheal adenoid cystic carcinoma: Adjuvant treatment outcome. Int J Clin Oncol 20: 686-692, 2015. 